\title{
Last Encounter Routing under Random Waypoint Mobility
}

\author{
Natasa Sarafijanovic-Djukic and Matthias Grossglauser \\ School of Computer and Communication Sciences \\ EPFL - Lausanne, Switzerland
}

\begin{abstract}
Last Encounter Routing (LER) algorithms for mobile ad hoc networks rely only on encounter histories at every node to route packets, and therefore do not need control traffic to track topology changes due to node mobility. LER exploits the fact that past information about a node's mobility helps to locate that node in the future. As we have pointed out in earlier work [1], the performance of LER algorithms depends on the mobility processes of nodes.

In this paper, we ask whether LER can work under the random waypoint (RWP) mobility model. This question is important for several reasons. First, as shown in [1], a good performance for the RWP model is harder to achieve than for another prominent mobility model, the random walk. This is because the RWP model has a much shorter relaxation time, i.e., a time-horizon over which past information is still useful. Also, the RWP model has a much less favorable ratio of number of encounters between nodes and the traveled distance. Second, in contrast to the random walk, the RWP model is predictable. This provides us with an opportunity to exploit additional information collected in an encounter (such as speed, direction, etc.) to improve routing.

We formally define the RWP model, and compute the optimal predictors for several observation sets, i.e., observed parameters of node mobility. We develop a new LER algorithm tuned to the RWP model called GREASE-RWP, and present simulation results that demonstrate that an efficient and scalable LER for the RWP model is possible.
\end{abstract}

\section{Introduction}

Last Encounter Routing (LER) $[1,2,3]$ refers to a type of routing algorithm where the destination of a packet is located without the help of a location service, and without any control traffic to track topology changes due to node mobility (except for local discovery packets to detect immediate neighbors). Rather, a packet is routed using only the encounter histories at nodes it is forwarded through. In its basic form, the encounter history consists of the time and location when a node was a directly connected neighbor of another node.

In [1], the following model was considered. A set of nodes perform independent random walks on a square lattice. Two nodes are directly connected neighbors if they reside at the same lattice point. Every node remembers when and where it has encountered every other node, in a last encounter table. A very 
simple algorithm called EASE was introduced in [1] to compute a route from a source node to a destination node, based only on LE history at every node. It was shown that the expected total cost of EASE routes is a small multiple of the expected shortest path length between a random source and destination. In other words, EASE is a scalable LER algorithm for the random walk mobility model, as the cost of routes relative to the shortest path does not blow up as the network size increases.

It is clear that the performance of LER algorithms is closely tied to the mobility pattern of the nodes in the network. To see this, consider an extreme scenario where there is no dependence between a node's position at different times, i.e., nodes "jump around" randomly in the network domain. In this case, history information is of no use, and any LER algorithm would perform as poorly as an exhaustive search for the destination.

The main question, then, is the interplay between the mobility model and the performance of LER algorithms. A complete answer to this question remains elusive, but it is possible to develop some intuition about features of the mobility model that are favorable to LER algorithms. In particular, we argue that the following three features of a mobility model help LER: locality, frequent intersections, and homogeneity. Locality means that a node's position at a time $t$ correlates with its position at a certain time $t+\tau$ in the future; this ensures that information about past encounters is actually useful in locating a node. Frequent intersections mean that a node over a given time interval tends to encounter a large number of other nodes. This ensures that information about that node's location in the LE tables of other nodes is refreshed frequently. Homogeneity means that the statistical properties of each node mobility are similar. This ensures that the speed of diffusion of encounter histories due to movements of other nodes is matched to that of a destination node. In this paper, all the scenarios we consider are homogeneous.

Based on these three features, we can see that the random walk mobility model is quite advantageous to LER. First, as a node performs independent steps over time, the dependence between the current and a future position of the node decreases only slowly with time. Specifically, it follows from the central limit theorem that the difference between the two positions $t$ seconds apart is a random variable with variance proportional to $\sqrt{t}$. Second, despite the locality, a node encounters other nodes frequently. Specifically, it was shown in [1] that over a time interval of length $t$, a node encounters $\Theta(t / \log t)$ other nodes. As a result, in the random walk mobility model, we observe that (i) a node's past location contains information about its current location over a relaxation time of $\Theta(n)$, where $n$ is the network size, and (ii) when a node moves by a distance $d$, it encounters approximately $\Theta\left(d^{2} / \log d\right)$ other nodes. This means that information about a destination node's movement is quite dense around this node, and that such an algorithm can rely on fairly old information to locate a destination.

In this paper, we consider LER for the random waypoint (RWP) mobility model. Informally, this model is defined as follows. Every node moves independently of any other node. A node selects a random waypoint uniformly in the area of the network, and moves towards this waypoint in a straight line and at 
constant speed. Once it reaches this waypoint, it selects a new waypoint independently of the previous one, and starts moving towards it (possibly after some pause time), and so forth.

There are several reasons why we are interested in LER for the RWP model. First, the model is well studied and is very prominent in simulation studies of mobile ad hoc networks. Second, it was shown in [1] that the LER algorithms EASE and GREASE did not perform well with this model. Third, given the discussion of helpful mobility features above, the RWP model is much less favorable to LER than the random walk.

To see this, let us perform a back-of-the-envelope comparison with the random walk. Note that the average transition time between waypoints is $\Theta(\sqrt{n})$, proportional to the diameter of the network. Once a node has gone through a waypoint, the dependence with its current position drops very rapidly. A past position of a node is therefore only useful for $\Theta(\sqrt{n})$ time. Furthermore, when a node moves by a distance $d$, it encounters only $\Theta(d)$ other nodes. This means that information about a destination node's movement is much less dense around this node, and that an LER algorithm must rely on fairly recent information to catch up with a destination.

On the other hand, the RWP model has a feature that is in our favor: the node movement is highly predictable over a short time-scale, because a node moves at constant speed on a straight line between waypoints. This provides us with an opportunity to route a packet towards the predicted current position of its destination, rather than simply towards the location of the encounter with the destination. This is not possible in the random walk case, where the best predictor for a node's future position is simply its current position.

In this paper, we introduce a version of the GREASE algorithm in [1] that is tuned to the specific features of the RWP mobility model. GREASE-RWP takes into account the short relaxation time of random waypoint mobility by reducing the encounter age it searches for initially to obtain the first estimate of the destination's location. It then uses mobility prediction as the packet approaches the destination in order to move towards the destination in "shortcuts". We evaluate GREASE-RWP through simulation in networks with up to 1000 nodes, and we find that GREASE-RWP has significantly better performance than GREASE, and seems to scale with network size. Therefore, LER can be efficient even for much less favorable mobility models than the random walk.

The paper is structured as follows. In Section 2, we formally define the RWP model and discuss some of its properties that matter in the context of LER. In Section 3, we present an LER algorithm specifically designed for the RWP model. We present simulation results in Section 4. Section 5 concludes the paper.

\section{The Random Waypoint Mobility Model}

In this section we formally define the RWP model, and discuss the properties that play an important role in the performance of LER.

Nodes move on a square torus of side $a$. The origin is in the center of the square. The vector $c-b$ is the vector on the torus surface such that 
$\|c-b\| \equiv d(b, c)$ is the shortest distance between the points $b$ and $c$. Nodes move independently of each other, so it is enough to define the movement of one node.

We consider a special case of the RWP model for simplicity. Specifically, we assume that nodes move with constant speed $v$, and that nodes do not pause at waypoints.

This RWP model is formally defined as follows.

- The speed $v$.

- the sequence of independently and identically distributed (i.i.d.) random variables $\left\{P_{w}\right\}_{w \in \mathbb{N}}$, uniformly distributed in the torus.

The $P_{w}$ is the $w$-th waypoint. If the node moves between the $w$-th and $w+1$-st waypoints we say that it is on the $w$-th segment. The distance between these waypoints $L_{w}=d\left(P_{w}, P_{w+1}\right)$ is the $w$-th segment length. The time for the node to traverse the $w$-th segment is the $w$-th segment duration. The time instant $S_{w}$ when the node is at the $w$-th waypoint is equal to:

$$
S_{w}=\sum_{j=1}^{w-1} \frac{L_{j}}{v},
$$

where $S_{1}=0$. The speed vector between the $w$-th and $w+1$-st waypoints is equal to:

$$
V_{w}=v \frac{P_{w+1}-P_{w}}{L_{w}} .
$$

Therefore, the node's position at time $t$ can be formally expressed as:

$$
X(t)=P_{w(t)}+V_{w(t)}\left(t-S_{w(t)}\right),
$$

where $w(t)$ is an index such that $t \in\left[S_{w(t)}, S_{w(t)+1}\right)$.

Note that on the torus the $L_{w}$ s are i.i.d, thus the random process $\left\{S_{w}\right\}_{w \in \mathbb{N}}$ is a renewal process. This property comes from the fact that torus represents isotropic space where all points are equivalent.

In the remainder of this section, we assume that time $t=0$ is the beginning of the observation period for the renewal process $\left\{S_{w}\right\}_{w \in \mathbb{N}}$ that has been operating long enough to be in steady state ${ }^{1}$. We enumerate the points of the renewal process as $\left\{S_{w}\right\}_{w \in \mathbb{Z}}$ with the convention that $S_{0} \leq 0<S_{1}$.

\subsection{Relaxation Time}

LER algorithms exploit a key property of realistic mobility processes, which is that the location of a node at a time $t$ and a time $t+\tau$ are dependent, and that therefore information collected through an encounter at time $t$ can be useful for a packet looking for its destination at time $t+\tau$. More specifically, consider

\footnotetext{
1 The RWP model with random speed does not possess a steady state in terms of average speed [5]. Here, this problem does not arise because of constant speed.
} 
a realistic mobility scenario where nodes have limited speed. Suppose that a source knows the destination position $X(t)$ and wants to route a packet to the destination at time $t+\tau$. If $\tau$ is relatively small, then the destination is in the small area around $X(t)$. Thus, information $X(t)$ is useful. If $\tau$ increases, then the area where the destination may be becomes larger and information $X(t)$ is less useful. For some large $\tau$, the destination may be anywhere in the network area and information $X(t)$ is useless.

The relaxation time $T_{r}$ is the minimum $\tau$ for which $X(t+\tau)$ does not depend on $X(t)$ any more (note that as we assume homogeneity, all nodes have the same relaxation time). The relaxation time is the maximum time after its observation that past information about a node's movement is still of use. It is therefore crucial to ensure that a LER algorithm does not rely on observations older than $T_{r}$, as this would lead to poor routes.

Let us consider the relaxation time in the RWP model. Let $Y_{t}=S_{w(t)+1}-t$ be the time the node travels to the next waypoint. After time $Y_{t}+L / v(L$ denotes the distribution of the segment lengths $\left.L_{w} s\right)$ the node will reach the second next waypoint. Hence, $X(t+\tau)$ for $\tau>Y_{t}+L / v$ depends only on $P_{w} \mathrm{~s}$, where $w \geq w(t)+2$. The $X(t)$ depends only on $P_{w(t)}$ and $P_{w(t)+1}$. Therefore, the positions $X(t)$ and $X(t+\tau)$ are independent for $\tau>Y_{t}+L / v$.

\subsection{Prediction}

In the RWP model, a node moves in a sequence of lines. This results in fewer encounters with other nodes relative to the traveled distance, and hence a low density of nodes with recent encounters around this node. On the other hand, this movement also implies excellent predictability of a node's position a certain time into the future, given its current location and other observations, such as direction and speed. Our goal is to use prediction of the destination position in order to decrease the routing cost of LER.

The observations available to a node on its own mobility process depend on the scenario. At one extreme, a node might be able to determine only some basic parameters about its instantaneous movement, such as its direction and speed. This might arise when a node's movement is subject to external influences, and if the node lacks the capability to identify waypoints. At the other extreme, a node might be able to know its precise movements a long time into the future. This might arise when a node's movement is predetermined by the node itself (e.g., a person running errands in a city, or a doctor visiting patients). Obviously, the predictability improves as the observation set increases. As our focus here is on the "tough" cases, we only consider observation sets that do not look beyond the next waypoint. Specifically, we calculate mobility predictors for the following three different observation sets $\theta$ :

1. the node's current position and speed vector;

2. the node's current position, speed vector and previous waypoint;

3. the node's current position, speed vector and next waypoint. 
The optimal mobility predictor is the one that minimizes in some sense the error between the node's predicted and true positions. We use the minimum mean square error criterion [7].

Definition 1. The minimum mean square error (MMSE) predictor of the node's position $X(t+\tau)$ given an observation $\theta(t)$ at time $t$ minimizes

$$
E\left[(X(t+\tau)-\widehat{X}(t+\tau))^{2} \mid \theta(t)\right] .
$$

and is given by

$$
\widehat{X}(t+\tau)=E[X(t+\tau) \mid \theta(t)] .
$$

We derive our predictors under the following assumptions. First, we assume that we make the observation at a random time $t$. Second, we neglect the direction the node is moving to. The length of the current interarrival interval is dependent on the direction; we ignore this for simplicity.

Theorem 1. The MMSE predictor $\widehat{X}(t+\tau)$ given the following observations:

- the node's current position $X(t)=x(t)$

- the node's current speed vector $V(t)=v(t)$

is given by:

$$
\widehat{x}(t+\tau)=x(t)+\frac{v(t)}{v}\left(P(Z \geq v \tau) v \tau+E\left[Z 1_{\{Z<v \tau\}}\right]\right),
$$

where $Z$ is a random variable with the pdf equal to:

$$
f_{Z}(z)= \begin{cases}c\left(1-\frac{z^{2} \pi}{a^{2}}\right) & \text { if } 0 \leq z \leq \frac{a}{2} \\ c\left(1-\frac{z^{2} \pi}{a^{2}}+\frac{4 z^{2}}{a^{2}} \arccos \frac{a}{2 z}-\frac{2}{a} \sqrt{\left.z^{2}-\frac{a^{2}}{4}\right)}\right. & \text { if } \frac{a}{2} \leq z \leq \frac{a \sqrt{2}}{2} \\ 0 & \text { otherwise }\end{cases}
$$

where $c=6(a \sqrt{2}+a \ln (1+\sqrt{2}))^{-1}$.

Theorem 2. The optimal mobility predictor of $X(t+\tau)$ given the following observations:

- the node's current position $X(t)=x(t)$

- the node's current speed vector $V(t)=v(t)$

- the node's previous waypoint $P_{w(t)}=p$

is given by:

$$
\widehat{x}(t+\tau)=x(t)+\frac{v(t)}{v}\left(P(Z \geq v \tau) v \tau+E\left[Z 1_{\{Z<v \tau\}}\right]\right),
$$

where $Z$ is a random variable with pdf

$$
f_{Z}(z)=\frac{f_{L}\left(z+l_{0}\right)}{1-F_{L}\left(l_{0}\right)}
$$

where $l_{0}=d(p, x(t))$, and $f_{L}(l)$ and $F_{L}(l)$ are the pdf and the cdf of the segment length (see Lemma 1 in the Appendix). 
Theorem 3. The optimal mobility predictor of $X(t+\tau)$ in the defined $R W P$ model if we know the following observations:

- the node's current position $X(t)=x(t)$

- the node's current speed vector $V(t)=v(t)$

- the node's next waypoint $P_{w(t)+1}=p_{\text {next }}$

is given by:

$$
\widehat{x}(t+\tau)=\left\{\begin{array}{ll}
x(t)+v(t) \tau & \text { if } v \tau \leq d\left(x(t), p_{\text {next }}\right) \\
p_{\text {next }} & \text { if } v \tau>d\left(x(t), p_{\text {next }}\right)
\end{array} .\right.
$$

Next, we give the main idea on how we derive the predictors. The details can be found in the Appendix. To calculate the conditional expectation of the $X(t+\tau)$ given the observation set $\theta(t)$, we express $X(t+\tau)$ as

$$
X(t+\tau)=X(t)+1_{\left\{\tau \leq Y_{t}\right\}} V(t) \tau+1_{\left\{\tau>Y_{t}\right\}}\left(V(t) Y_{t}+\triangle P\left(\tau-Y_{t}\right)\right),
$$

where $Y_{t}=S_{w(t)+1}-t$ is the time until the node hits the next waypoint, and $\triangle P\left(\tau-Y_{t}\right)$ is the displacement of the node after reaching the next waypoint until time $t+\tau$. Figure 1 explains (8). Expected value of the $\triangle P\left(\tau-Y_{t}\right)$ is equal to zero (Lemma 2 in the Appendix). Thus, we need to calculate only the conditional expectation of the $Y_{t}$ given the $\theta(t)$. Since we observe the system at a random point in time, the $Y_{t}$ is the residual time of the renewal process $\left\{S_{w}\right\}_{w \in \mathbb{N}}$. Note that the segment duration on which the node is at a random time $t$ is not distributed according to the distribution of the intervals $S_{w+1}-S_{w}$. This is because a long segment is more likely to be "intercepted" by our observation than a short one [6]. We use elements of Palm calculus (Chapter 12,[8]) to relate these different viewpoints and to calculate the distribution of the residual time in the concrete case. Since the calculation for Theorem 3 is trivial, we give further calculations only for Theorems 1 and 2 in the Appendix.

We are unable to calculate the mobility predictors in Theorems 1 and 2 in a closed form. Instead, we use approximations to derive good closed-form predictor (cf. Approximations 1 and 2 in the Appendix).

\section{A LER Algorithm for the Random Waypoint Model}

We now introduce a new LER algorithm that takes into account the features of the RWP model described in the previous section. We first define some notation. Nodes are indexed by $1,2, \ldots, n$ where $n$ is the number of nodes. We focus on a single destination node with index 1 and assume w.l.g. that a packet is sent to the destination at time $t=0$. The $X_{i}(t)$ is the position of the node $i$ at time $t$. The $T_{i}(t)$ is the age of last encounter between node $i$ and the destination. The $\theta_{i}(t)$ is the observation set of the node $i$ at time $t$. The optimal mobility predictor of the destination position at time $t$ is denoted by $\widehat{X_{1}}(t)$. Recall that $T_{r}=\Theta(\sqrt{n})$ is the relaxation time. 


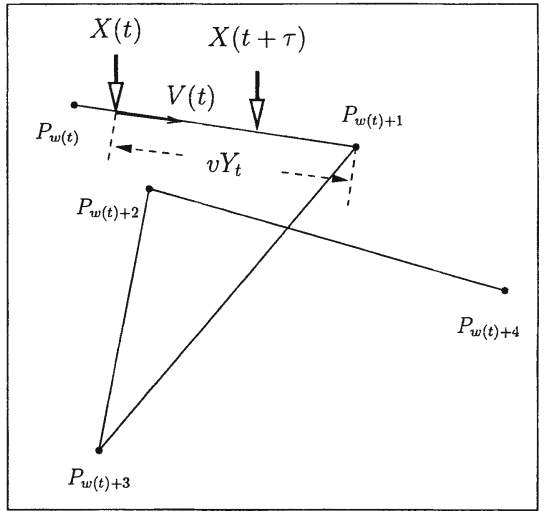

$\tau \leq Y_{t}$

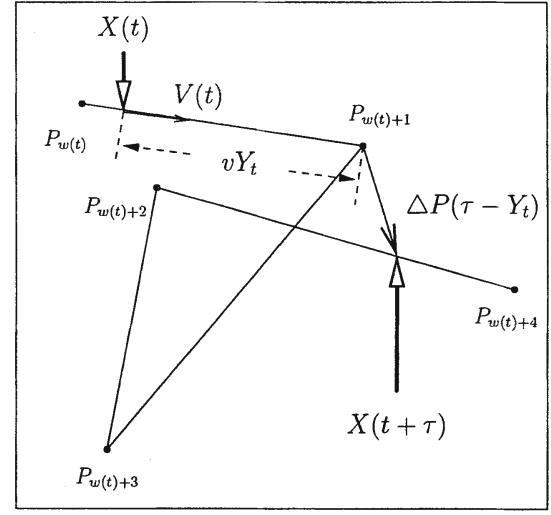

$\tau>Y_{t}$

Fig. 1. Explanation of (8): one possible movement of a node and its positions at times $t$ and $t+\tau$ for different values of $\tau$.

\section{Algorithm 1: GREASE-RWP}

1 Set $Y_{0}:=X_{s}(0), T_{0}:=E\left[T_{r}\right], k:=0$.

2 Repeat

3 Search the nodes around $Y_{k}$ in the order of increasing distance until a node $i$ is found such that $T_{i}(0)<T_{k}$.

4 Let $T_{k+1}=T_{i}(0)$, and $Y_{k+1}:=\widehat{X_{1}}(0)=f\left(\theta_{1}\left(-T_{k+1}\right)\right)$ be the new anchor point.

$5 \quad$ While not at $Y_{k+1}$

6 Route packet: find next hop $j$ towards $Y_{k+1}$ and forward packet to $j$.

$7 \quad$ If $T_{j}(0)<T_{k+1}$, then $T_{k+1}:=T_{j}(0), Y_{k+1}:=\widehat{X_{1}}(0)=f\left(\theta_{1}\left(-T_{k+1}\right)\right)$.

8 End while

$9 \mathrm{k}++$.

10 Until $Y_{k}=X_{1}(0)$.

The main new features in GREASE-RWP in comparison with GREASE [1] are a more aggressive initial search and the use of prediction.

Aggressive initial search means that a source searches for an encounter with the destination younger than average relaxation time. As we saw in Sect. 2.1 average relaxation time is of the order of a segment time. This means that the packet immediately goes to the few last segments of the destination movement. Thus the packet avoids the useless walking over the network area.

Prediction is incorporated into GREASE by sending a packet towards the predicted position of the destination rather than towards the location of the encounter. Thus the packet tends to take "shortcuts" from segment to segment.

Of course, the use of prediction requires nodes to remember additional statistics about other nodes, in addition to the position of last encounter. This is incorporated into LER as follows. Every node tracks its own mobility. When two 
nodes encounter each other, they exchange observations about their own mobility (e.g., their current speed and direction) along with the "hello" messages. Each node records these observations in its LE table (cf. Fig. 2). They are used to calculate the optimal mobility predictor.

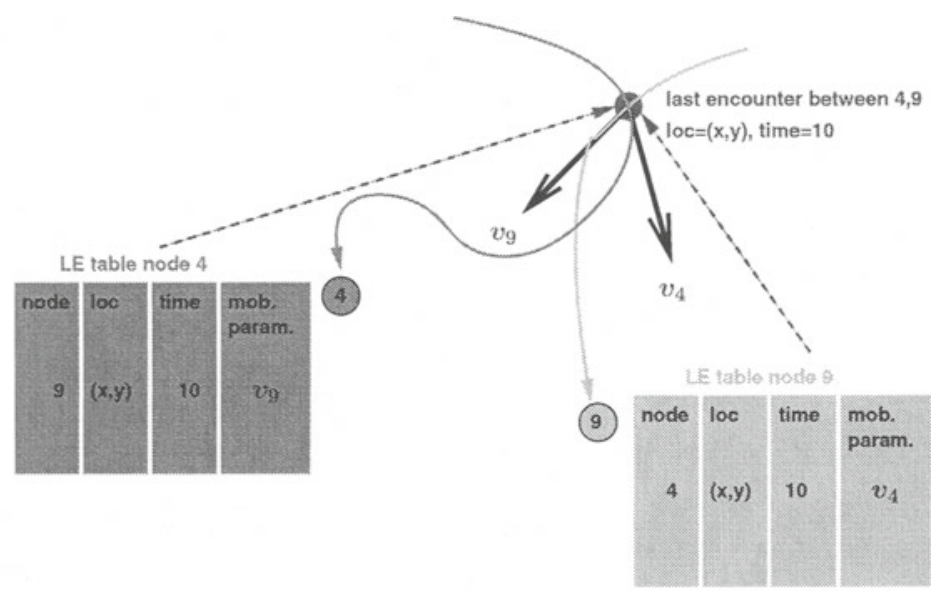

Fig. 2. A last encounter table in every node remembers both the location and time of the last encounter with every other node in the network, and in addition, some observations about every other node's movement at the time of the last encounter (in this example, speed vector).

\section{Simulation Results}

We have performed extensive simulations to evaluate the efficiency and scalability of GREASE-RWP. By efficiency, we mean the average routing cost relative to the shortest path cost. By scalability, we mean how the efficiency scales with increasing the network size.

Nodes move on a torus of surface $n$ according to the RWP model. Initially the nodes are placed uniformly in the torus. We let them to move for a sufficient warm-up period so that a fair proportion of node pairs have encountered at least once. Then, we assume that the nodes are frozen for the time of the routing of the packet.

Routing of the packet is performed through the GREASE (Algorithm 2 in [1]), GREASE-RWP (Algorithm 1 in this paper), GREASE-M and GREASE-A algorithms. GREASE-M is GREASE with mobility prediction. We obtain it by changing the step 4 in GREASE with the step 4 in GREASE-RWP. GREASE-A is GREASE with aggressive initial search. We obtain it by changing the step 1 in GREASE with the step 1 in GREASE-RWP. The $M_{i}(i=1,2,3)$ denotes respectively the predictors from Theorems 1-3. If nothing is specified the predictor M2 is used. 
At every time $t$, we assume that connectivity is given by the Delaunay graph generated by the set of points $\left\{X_{i}(t)\right\}$. This is equivalent to generating the Voronoi tessellation of the set of points $\left\{X_{i}(t)\right\}$, such that every node $X_{i}(t)$ is the center of a Voronoi cell, and is connected to the center nodes of its adjacent cells. Each node updates the entries in its LE table for its directly connected neighbors.

The advantage of this topology over other topologies (e.g., $k$ nearest neighbors) is that we are guaranteed that a node always has a neighbor that is closer to the destination (except when that destination is already in the first node's Voronoi cell). Therefore, a packet can always make progress towards its anchor point, and we do not have to deal with backtracking, avoiding routing loops, etc., which are not the focus of this work. This allows us to focus on the main issue at hand, i.e., the quality of routes based on diffused information about last encounters.

The main metric we evaluate is the relative cost of the routes compared with the cost of the shortest path route. This metric therefore captures the relative penalty incurred for not having the exact position of the destination available.

Figure 3 shows an example of a route computed by GREASE and GREASERWP for the same source- destination pair. We see that GREASE-RWP achieves the shorter route than GREASE by increasing the cost of the initial search and by taking shortcuts with the help of mobility prediction.

In Fig. 4(a) and 4(b) we give the relative cost conditional on the distance between the source and destination. This provides an indication whether the relative quality of the routes increases or decreases as the routes get longer.

Figure 4(a) shows the benefit of mobility prediction if different observation sets are used. If more information about node mobility is available, improvement of GREASE is better.

Figure 4(b) shows that the penalty of the GREASE-RWP algorithm because of the uncertainty of the destination location is only 2.5 times greater than the shortest route (i.e., the ideal case where the destination location is known). Also, it shows that aggressive initial search or mobility prediction alone significantly increase efficiency.

In Fig. 5 we give dependence of the average relative cost of routes on the number of nodes $n$. This provides an indication whether the relative cost of the routes is scalable with the network size.

\section{Discussion and Conclusion}

In this paper, we have shown that efficient and scalable last encounter routing under random waypoint mobility is possible. We have achieved this by devising a new instance of the GREASE algorithm, which differs from the version reported in [1] in two respects. First, we have exploited the inherent predictability of nodal movement in the RWP model over short time-scales. When a packet looking for its destination picks up a more recent encounter, it can compute a predicted location for the destination that is better on average than the location of that encounter itself. Second, we account for the fact that the RWP model has a very 


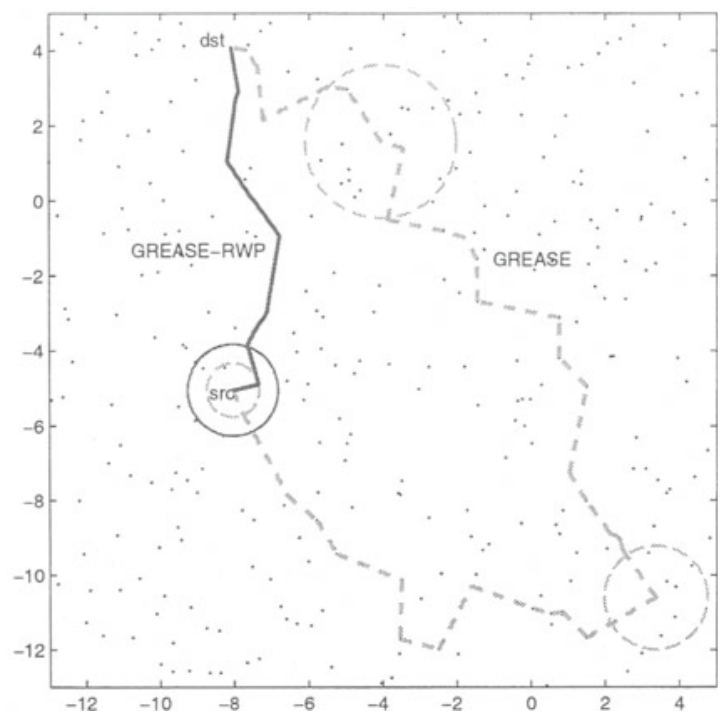

Fig. 3. A sample route computed by GREASE and GREASE-RWP for the same source-destination pair.

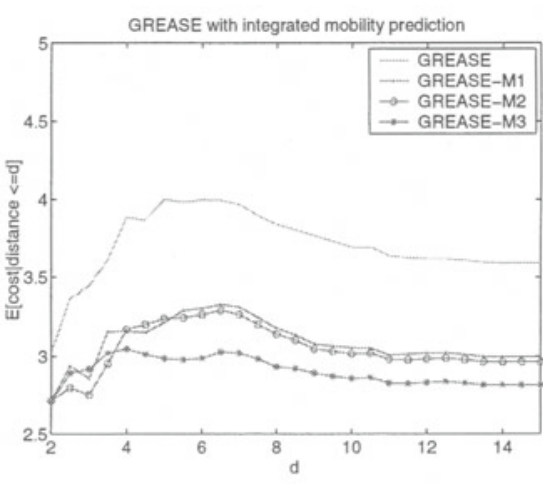

(a)

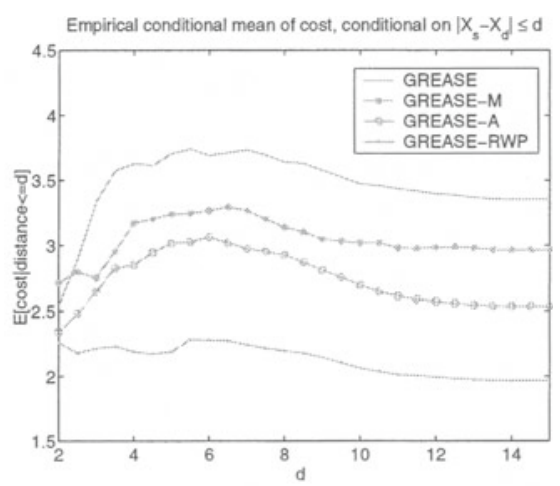

(b)

Fig. 4. a) Impact of the different observation sets in mobility prediction on the efficiency of GREASE. b) Efficiency of GREASE, GREASE-M (mobility prediction), GREASE-A (aggressive initial search) and GREASE-RWP (mobility prediction and aggressive initial search).

short relaxation time, by forcing a low target age for the initial search. Thus the packet avoids using outdated past encounters that are independent of the destination's actual position.

We have performed simulations that show the following results. First, the GREASE-RWP algorithm achieves drastically better performance than the "non-RWP" version reported in [1]. Specifically, the total average route cost 


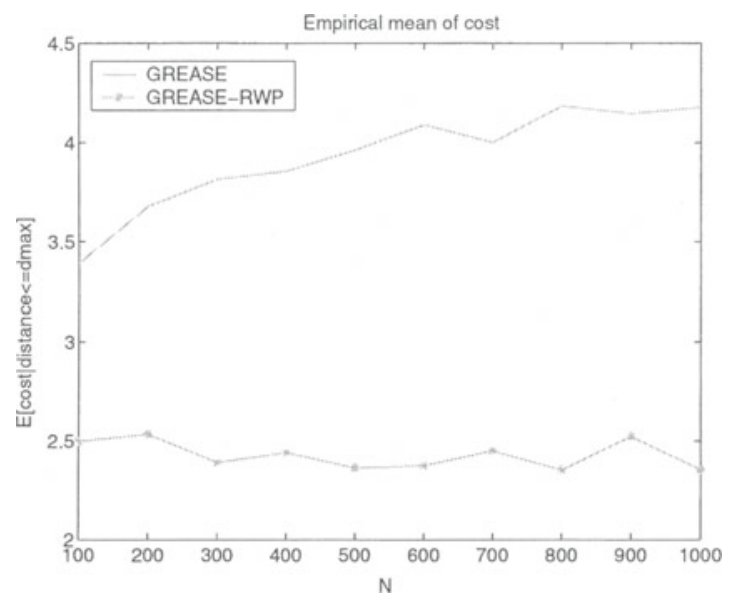

Fig. 5. Scalability of the GREASE-RWP algorithm.

is slightly more than twice as long as the shortest path. This is quite remarkable, given that no resources were invested to track the rapid change in the network topology due to RWP mobility. Second, our results show, as we would expect, that the benefit of prediction depends on what observations are available about a node's mobility. It should be pointed out that if we extended prediction beyond the next waypoint, the performance would be further improved. Third, the cost of GREASE-RWP routes relative to the shortest path does not seem to increase as we scale up the network size $n$. Therefore, we believe that a similar scaling result as shown in [1] for the random walk holds for the RWP model as well.

Although this paper shows that efficient and scalable LER is possible beyond the random walk mobility model, we are not yet able to fully characterize the space of mobility models for which this is true. However, as the RWP model can be considered unfavorable to LER, our results suggest that LER may be applicable to a large class of mobility processes. In our future work, we plan to find more general necessary conditions for a mobility process to be feasible.

Another open question concerns the generality of particular instances of LER algorithms. Note that GREASE-RWP would perform poorly with the random walk model, because the initial search criterion would be too aggressive; whereas, the original GREASE described in [1] has been shown to perform relatively poorly for the RWP model. Our ultimate goal, of course, would be to devise a LER algorithm that is universal, i.e., that would perform well over the entire class of feasible mobility models. We conjecture that such a model would require some a-priori knowledge about the mobility process, as it would be essentially impossible otherwise to decide what initial age to search for. Such a-priori knowledge might in turn be obtained through some estimation process that would run in the background as an "outer loop" (e.g., through a gossiping protocol) and make estimated mobility parameters available at every node. 


\section{References}

1. M. Grossglauser and M. Vetterli. Locating Nodes with EASE: Last Encounter Routing through Mobility Diffusion. IEEE Infocom, April 2003, San Francisco, CA.

2. H. Dubois-Ferrière, M. Grossglauser and M. Vetterli. Age Matters: Efficient Route Discovery in Mobile Ad Hoc Networks Using Encounter Ages. In Proc. ACM MOBIHOC 03, Maryland, June 2003.

3. H. Dubois-Ferrière, M. Grossglauser and M. Vetterli. Space-Time Routing in Ad Hoc Networks, Ad Hoc Now 03, MontrÃ(C)al, Canada, October 2003.

4. David B. Johnson and David A. Maltz. Dynamic Source Routing in Ad Hoc Wireless Networks. In Mobile Computing, edited by Tomasz Imielinski and Hank Korth, Chapter 5, pages 153-181, Kluwer Academic Publishers, 1996.

5. J. Yoon, M. Liu and B. Noble. Random Waypoint Considered Harmful. In Proc. IEEE INFOCOM, vol 2, pp 1312-1321, April 2003, San Francisco, CA.

6. L. Kleinrock. Queueing Systems, Volume 1, Jhon Wiley \& Sons, 1975.

7. R. Gray and L. Davisson. An Introduction to Statistical Signal Processing. http://www-ee.stanford.edu/ gray/sp.html

8. Jean-Yves Le Boudec. Performance Evaluation Lecture Notes. EPFL, 2003. http://ica1www.epfl.ch/perfeval/lectureNotes

\section{Appendix}

\section{Auxiliary Results}

Lemma 1. The sequence of the segment lengths $L_{w} s$ has the following pdf:

$$
f_{L}(l)=\left\{\begin{array}{ll}
\frac{2 l \pi}{a^{2}} & \text { if } 0 \leq l \leq \frac{a}{2} \\
\frac{2 l \pi}{a^{2}}-\frac{8 l}{a^{2}} \arccos \frac{a}{2 l} & \text { if } \frac{a}{2} \leq l \leq \frac{a \sqrt{2}}{2} \\
0 & \text { otherwise }
\end{array} .\right.
$$

Lemma 2. The expected displacement of a node from a waypoint is equal to zero, i.e., the expectation of the random variable $X\left(S_{w}+\triangle t\right)-X\left(S_{w}\right)$ is equal to zero, for every $w$ and $\triangle t>0$.

\section{Optimal Predictors}

Proof of Theorems 1 and 2. Using (2) and (8), the optimal mobility predictor is:

$$
\begin{aligned}
\hat{x}(t+\tau)= & E[X(t+\tau) \mid \theta(t)]= \\
= & x(t)+v(t) \tau P\left(Y_{t} \geq \tau \mid \theta(t)\right)+v(t) E\left[Y_{t} 1_{\left\{Y_{t}<\tau\right\}} \mid \theta(t)\right]+ \\
& +E\left[\triangle P\left(\tau-Y_{t}\right) 1_{\left\{Y_{t}<\tau\right\}} \mid \theta(t)\right] .
\end{aligned}
$$

The last term of this equation is equal to 0 (Lemma 2). Thus, we obtain:

$$
\hat{x}(t+\tau)=x(t)+v(t) \tau P(Z \geq v \tau)+\frac{v(t)}{v} E\left[Z 1_{\{Z>v \tau\}}\right]
$$


where $Z$ is the random variable with the same distribution as $v Y_{t}$ given $\theta(t)$. Next, we calculate the distribution of $Z$ separately for Theorems 1 and 2 .

In Theorem 1, the set of observations is $\theta(t)=\{X(t)=x(t), V(t)=v(t)\}$. The residual time $Y_{t}$ depends only on the renewal process $S_{w}$. The $S_{w}$ s depend only on the segment lengths $L_{w}$ s because of the constant speed. The $L_{w}$ s do not depend on $X(t)$ since every point in the torus is the same. Therefore, the $Y_{t}$ does not depend on $X(t)$. As mentioned previous, we neglect that the $Y_{t}$ depends on the direction of the speed vector, thus the $Y_{t}$ does not depend on $V(t)$. Hence, the $Z$ has the same distribution as $v Y_{t}$. The pdf of residual time $Y_{t}$ is equal to [6]:

$$
f_{Y}(y)=\frac{1-F_{R}(y)}{m_{R}},
$$

where $F_{R}(x)$ is the cdf of the intervals $R_{w}=S_{w+1}-S_{w}$ and $m_{R}$ is the mean of $R_{w}$. Since $R_{w}=L_{w} / v$, the pdf of $Z$ is:

$$
f_{Z}(z)=\frac{1-F_{L}(z)}{m_{L}},
$$

where $F_{L}(z)$ is the cdf of $L_{w} \mathrm{~s}$ (Lemma 1$)$, and $m_{L}=a / 6(\sqrt{2}+\ln (1+\sqrt{2}))$ is its expected value. We obtain the pdf of $Z$ given by (4).

In Theorem 2, the set of observations is $\theta(t)=\{X(t)=x(t), V(t)=$ $\left.v(t), P_{w(t)}=p\right\}$. As in the previous case, the residual time $Y_{t}$ does not depend on $X(t)$ and $V(t)$. Knowing both $X(t)=x(t)$ and $P_{w(t)}=p$ we know that the previous waypoint was at time $t-d(p, x(t)) / v=t-t_{0}$. This means that there is a point at time $t-t_{0}$ and that there is no point in interval $\left(t-t_{0}, t\right)$. This second condition is equivalent to $Y_{t-t_{0}} \geq t_{0}$. If we denote the conditional probability given that there exists a point at time $t$ as $P^{t}$ then the cdf of $Z$ is equal to:

$$
\begin{aligned}
P(Z \leq z) & =P\left(v Y_{t} \leq z \mid X(t)=x(t), V(t)=v(t), P_{w(t)}=p\right) \\
& =P\left(v Y_{t} \leq z \mid \text { there exists a point at time } t-t_{0}, Y_{t-t_{0}} \geq t_{0}\right) \\
& =P^{t-t_{0}}\left(v Y_{t} \leq z \mid Y_{t-t_{0}} \geq t_{0}\right)=P^{0}\left(Y_{0} \leq \frac{z}{v}+t_{0} \mid Y_{0} \geq t_{0}\right) \\
& =P^{0}\left(S_{1} \leq \frac{z}{v}+t_{0} \mid S_{1} \geq t_{0}\right) .
\end{aligned}
$$

Using the result of Palm calculus that $P^{0}\left(S_{1} \leq x\right)=P^{0}\left(R_{1} \leq x\right)=F_{R}(x)$ (Chapter 12,[8]), we obtain:

$$
P(Z \leq z)=\frac{F_{R}\left(\frac{z}{v}+t_{0}\right)-F_{R}\left(t_{0}\right)}{1-F_{R}\left(t_{0}\right)} .
$$

Since $R_{w}=L_{w} / v$, the cdf of $Z$ can be expressed as:

$$
P(Z \leq z)=\frac{F_{L}\left(z+d_{0}\right)-F_{L}\left(d_{0}\right)}{1-F_{L}\left(d_{0}\right)},
$$

and we obtain the pdf of $Z$ given by (6). 
Approximation 1. We are unable to compute in a closed form the predictor in Theorem 1 . Therefore, we use the following mobility predictor:

$$
\begin{aligned}
& \widehat{x}(t+\tau)= \begin{cases}x(t)+\frac{v(t)}{v}\left(v \tau-\frac{c(v \tau)^{2}}{2}+\frac{c \pi(v \tau)^{4}}{12 a^{2}}\right) & \text { if } 0 \leq v \tau \leq \frac{a}{2} \\
x(t)+\frac{v(t)}{v} E[Z] & \text { if } v \tau>\frac{a \sqrt{2})}{2}\end{cases} \\
& \widehat{x}(t+\tau) \approx x(t)+\frac{v(t)}{v} E[Z] \text { if } \frac{a}{2} \leq v \tau \leq \frac{a \sqrt{2}}{2} .
\end{aligned}
$$

The mean value of the $Z$ is given by:

$$
E[Z]=\frac{E[L]}{2}+\frac{\operatorname{Var}(L)}{2 E[L]}
$$

where the mean $E[L]$ and the variance $\operatorname{Var}(L)$ of the $L_{i}$ s are equal to $E[L]=$ $a / 6(\sqrt{2}+\ln (1+\sqrt{2}))$ and $\operatorname{Var}(L)=\frac{a^{2}}{6}-E[L]^{2}$, respectively. We make an approximation in the region $\frac{a}{2}<v \tau<\frac{a \sqrt{2}}{2}$ by neglecting the probability that there is not any waypoint in the period of $v \tau$.

Approximation 2. We are unable to compute in a closed form the predictor in Theorem 2. Therefore, we use the following mobility predictor:

$$
\begin{aligned}
& \widehat{x}(t+\tau)=x(t)+\frac{v(t)}{v} \frac{\pi l_{0}^{3}-\pi\left(v \tau+l_{0}\right)^{3}+3 v \tau a^{2}}{3\left(a^{2}-l_{0}^{2} \pi\right)} \text { if }\left(l_{0}, v \tau+l_{0}\right) \in\left[0, \frac{a}{2}\right]^{2} \\
& \widehat{x}(t+\tau) \approx \begin{cases}x(t)+v \tau & \text { if } 0 \leq l_{0} \leq \frac{a}{2}, \frac{a}{2}-l_{0} \leq v \tau \leq E[Z] \\
x(t)+E[Z] & \text { if } 0 \leq l_{0} \leq \frac{a}{2}, \frac{a}{2}-l_{0} \leq v \tau, v \tau>E[Z] . \\
x(t) & \text { if } l_{0}>\frac{a \sqrt{(2)}}{2}\end{cases}
\end{aligned}
$$

The mean value of the $Z$ is given by:

$$
E[Z]=\frac{3 a^{2}\left(E[L]-l_{0}\right)+\pi l_{0}^{3}}{3\left(a^{2}-l_{0}^{2}\right)}
$$

where the mean value of the $L_{i} \mathrm{~S}$ is equal to $E[L]=\frac{a}{6}(\sqrt{2}+\ln (1+\sqrt{2}))$. We make an approximation in the region $0 \leq l_{0} \leq \frac{a}{2}, \frac{a}{2}-l_{0} \leq v \tau \leq E[Z]$ by neglecting probability that there is a waypoint in the period of $v \tau$. We make also an approximation in the region $0 \leq l_{0} \leq \frac{a}{2}, \frac{a}{2}-l_{0} \leq v \tau, v \tau>E[Z]$ by neglecting probability that there is not any waypoint in the period of $v \tau$. In the region $l_{0}>a / 2$ we do not make mobility prediction because we are unable to compute $E[Z]$. The probability of the appearance of this last case during the operation of the LER algorithm is small because the probability that $l_{0}>a / 2$ is small. Thus, this will have a small effect on the predictor. 